\title{
On the continuity of the free boundary in some class of two-dimensional problems
}

\author{
MICHEL CHIPOT ${ }^{\dagger}$ \\ Institut für Angewandte Mathematik, Universität Zürich, 8057 Zürich,
}

[Received 14 October 1999 and in revised form 27 July 2000]

We introduce a new method to show the continuity of the free boundary for problems in dimension two.

\section{Introduction}

A free boundary is a curve or a surface splitting a domain where a phenomenon is taking place into two subsets. Its position is an unknown of the problem at hand, as are the two subsets. To determine them one of the modern ideas on free boundary problems has been to introduce as unknown the characteristic function of one of them. The other should then be the complement. However, looking for the characteristic function of a set is like looking for the set itself and this does not make the task much simpler. Developing this idea further leads to enlarging the class of functions in which should be found this characteristic function, for instance to a subset of functions bounded between 0 and 1 -see [5]. If the existence of a solution to the problem becomes easier to establish a delicate task consists in showing that the function found is really a characteristic function and to see if the set characterized by this function and its complement are separated by a curve. This is what we would like to address here for some class of problems. Let us now introduce a general setting that will fit then several applications.

Let $\Omega$ denote a piecewise smooth cylindrical open set of $\mathbb{R}^{2}$.

More precisely, denote by $\gamma, \bar{\gamma}$ two functions from $I=(a, b)$ into $\mathbb{R}$ and set

$$
\begin{aligned}
\Omega & =\left\{\left(x_{1}, x_{2}\right) \in \mathbb{R}^{2} \mid x_{2} \in(a, b), \quad \underline{\gamma}\left(x_{2}\right)<x_{1}<\bar{\gamma}\left(x_{2}\right)\right\}, \\
\Gamma_{1} & =\left\{\left(\underline{\gamma}\left(x_{2}\right), x_{2}\right), x_{2} \in I\right\}, \\
\Gamma_{3} & =\left\{\left(\bar{\gamma}\left(x_{2}\right), x_{2}\right), x_{2} \in I\right\}, \\
\Gamma_{2} & =\Gamma \backslash \Gamma_{1} \cup \Gamma_{3},
\end{aligned}
$$

where $\Gamma$ denotes the boundary of $\Omega$.

Let $K(x)=K=\left(k_{i j}\right)$ be a two-by-two matrix with

$$
k_{i j} \in L^{\infty}(\Omega) \quad \forall i, j=1,2,
$$

and let

$$
h \in L^{\infty}(\Omega)
$$

\footnotetext{
†Email: chipot@amath.unizh.ch
} 
denote a bounded function.

Let us introduce the following class of free boundary problems. We look for a couple $(u, \chi)$ such that it holds that

$$
\left\{\begin{array}{l}
(u, \chi) \in H^{1}(\Omega) \times L^{\infty}(\Omega), 0 \leqslant \chi \leqslant 1 \text { a.e. in } \Omega, \\
u \geqslant 0 \text { a.e. in } \Omega, \quad \chi=1 \text { on }[u>0], \\
\int_{\Omega}\left\{K(\nabla u) \cdot \nabla z+h \chi z_{x_{1}}\right\} \mathrm{d} x \leqslant 0 \quad \forall z \in H^{1}(\Omega), \quad z=0 \text { on } \Gamma_{1} \cup \Gamma_{2}, \quad z \geqslant 0 \text { on } \Gamma_{3} .
\end{array}\right.
$$

Here $K(\nabla u)$ denotes the vector obtained by applying $K$ to the vector $\nabla u,[u>0]=\{x \in \Omega \mid$ $u(x)>0\}$ : see $[4,10,12]$ for an introduction and the notation on Sobolev spaces.

We present a two-dimensional technique allowing us to show the continuity of the free boundary of such a problem. By free boundary we mean, roughly speaking, the set

$$
\overline{[u>0]} \cap \overline{[u=0]}
$$

where $\bar{A}$ stands for the closure of a set and $[u=0]=\{x \in \Omega \mid u(x)=0\}$. The set defined by (1.8) could be empty but a more precise definition of the free boundary will be given below.

Such a problem - as we will see in the last section of the paper-arises in various contexts: for instance, the dam problem (see [1,2,5-9]) or problems in aluminium electrolysis [3, 13]. It also should give new results in the framework of the two-dimensional continuous casting Stefan problem or in lubrication theory see [14].

In Section 2 we show, under some assumptions on $K$ and $h$, that the free boundary (1.8) can be described by a lower semicontinuous function. In Section 3 we show that this function is continuous. Then, as already mentioned above, we give some applications.

\section{Lower semicontinuity of the free boundary}

Let us start with some preliminary results regarding problem (1.7). Let us assume in this section that

$$
\begin{gathered}
K(x)(\xi) \cdot \xi \geqslant \underline{K}|\xi|^{2} \quad \forall \xi \in \mathbb{R}^{2}, \quad \text { a.e. } x \in \Omega, \\
h \in H^{1}(\Omega), \quad h_{x_{1}} \geqslant 0, \\
h \geqslant \underline{h}>0 \quad \text { a.e. on } \Omega .
\end{gathered}
$$

We have denoted by $h_{x_{1}}$ the partial derivative of $h$ in the distributional sense and by $\underline{h}, \underline{K}$ positive constants. Then the following proposition holds.

Proposition 2.1 Let us assume that (2.1)-(2.3) hold. Let $u$ be a solution to (1.7). Then one has

$$
\nabla \cdot(K(\nabla u))+(h \chi)_{x_{1}}=0 \quad \text { in } \Omega,
$$

in the distributional sense: i.e. in $\mathcal{D}^{\prime}(\Omega)$. Moreover, one has also

$$
\begin{gathered}
\nabla \cdot(K(\nabla u))+\chi_{[u>0]} h_{x_{1}} \geqslant 0 \quad \text { in } \Omega, \\
\chi_{x_{1}} \leqslant 0 \quad \text { in } \Omega,
\end{gathered}
$$

in the distributional sense. 
$\left(\nabla \cdot v\right.$ denotes the divergence of the vector $v, \chi_{[u>0]}$ denotes the characteristic function of the set $[u>0]$.)

Proof. To prove (2.4) it is enough to consider $z \in \mathcal{D}(\Omega)$ in (1.7)—recall that $\mathcal{D}(\Omega)$ denotes the set of $C^{\infty}$-functions with compact support in $\Omega$ and $\pm z$ is a test function for (1.7).

To prove (2.5), for $\delta>0$, denote by $H_{\delta}$ an approximation of the Heaviside function, i.e. the function defined by

$$
H_{\delta}(x)= \begin{cases}1 & \text { for } \quad x \geqslant \delta, \\ x / \delta & \text { for } 0 \leqslant x \leqslant \delta, \\ 0 & \text { for } x \leqslant 0 .\end{cases}
$$

Then, for $\xi \in \mathcal{D}(\Omega), \xi \geqslant 0$ it is clear that $\pm H_{\delta}(u) \xi$ is a test function for (1.7). Thus we obtain

$$
\int_{\Omega}\left\{K(\nabla u) \cdot \nabla\left(H_{\delta}(u) \xi\right)+h \chi\left(H_{\delta}(u) \xi\right)_{x_{1}}\right\} \mathrm{d} x=0,
$$

which leads to

$$
\int_{\Omega}\left\{H_{\delta}(u) K(\nabla u) \cdot \nabla \xi+H_{\delta}^{\prime}(u) \xi K(\nabla u) \cdot \nabla u+h\left(H_{\delta}(u) \xi\right)_{x_{1}}\right\} \mathrm{d} x=0,
$$

(we used the fact (see (1.7)) that $\chi=1$ on $[u>0]$ ).

From (2.1) one deduces then that (recall that $H_{\delta}^{\prime} \geqslant 0$ )

$$
\int_{\Omega}\left\{H_{\delta}(u) K(\nabla u) \cdot \nabla \xi+h\left(H_{\delta}(u) \xi\right)_{x_{1}}\right\} \mathrm{d} x \leqslant 0 .
$$

Since $\xi \in \mathcal{D}(\Omega)$ one has clearly

$$
\int_{\Omega}\left\{h\left(H_{\delta}(u) \xi\right)_{x_{1}}+h_{x_{1}} H_{\delta}(u) \xi\right\} \mathrm{d} x=\int_{\Omega}\left(h H_{\delta}(u) \xi\right)_{x_{1}}=0
$$

from which one deduces

$$
\int_{\Omega}\left\{H_{\delta}(u) K(\nabla u) \cdot \nabla \xi-H_{\delta}(u) h_{x_{1}} \xi\right\} \mathrm{d} x \leqslant 0 .
$$

Letting $\delta$ go to 0 one obtains

$$
\int_{\Omega}\left\{K(\nabla u) \cdot \nabla \xi-\chi_{[u>0]} h_{x_{1}} \xi\right\} \mathrm{d} x \leqslant 0 \quad \forall \xi \in \mathcal{D}(\Omega), \quad \xi \geqslant 0 .
$$

This is precisely (2.5).

To get (2.6) one has by (2.4), (2.5)

$$
\chi_{[u>0]} h_{x_{1}}-(h \chi)_{x_{1}} \geqslant 0
$$

in the distributional sense. That is to say, for any $\xi \in \mathcal{D}(\Omega), \xi \geqslant 0$ it holds that

$$
\int_{\Omega} \chi_{[u>0]} h_{x_{1}} \xi \mathrm{d} x+\int_{\Omega} h \chi \xi_{x_{1}} \mathrm{~d} x \geqslant 0 .
$$


Using the fact that

$$
h \xi_{x_{1}}=(h \xi)_{x_{1}}-h_{x_{1}} \xi
$$

we obtain

$$
\int_{\Omega}\left\{\chi[u>0] h_{x_{1}} \xi-\chi h_{x_{1}} \xi\right\} \mathrm{d} x+\int_{\Omega} \chi(h \xi)_{x_{1}} \mathrm{~d} x \geqslant 0 .
$$

Since $\chi=1$ on $[u>0]$ we get

$$
\int_{\Omega} \chi(h \xi)_{x_{1}} \mathrm{~d} x \geqslant \int_{\Omega} \chi \cdot \chi_{[u=0]} h_{x_{1}} \xi \mathrm{d} x \geqslant 0
$$

by (2.2). Thus we arrive at

$$
\int_{\Omega} \chi(h \xi)_{x_{1}} \mathrm{~d} x \geqslant 0 \quad \forall \xi \in \mathcal{D}(\Omega), \quad \xi \geqslant 0 .
$$

By density this holds for any $\xi \in H_{0}^{1}(\Omega), \xi \geqslant 0$-replacing there $\xi$ by $\xi / h$ one gets

$$
\int_{\Omega} \chi \xi_{x_{1}} \mathrm{~d} x \geqslant 0 \quad \forall \xi \in \mathcal{D}(\Omega), \quad \xi \geqslant 0 .
$$

This completes the proof of Proposition 2.1.

COROLlary 2.2 Under the assumption (2.1) it holds for $u$ solution to (1.7) that

$$
u \in C(\Omega)
$$

i.e. $u$ is a continuous function in $\Omega$.

Proof. This follows from (2.4) and, for instance, [10: Proposition 6, p. 586].

REMARK 2.3 As a consequence of Corollary 2.2 the set

$$
[u>0]=\{x \in \Omega \mid u(x)>0\}
$$

is open in $\Omega$.

COROLlary 2.4 Let us assume that (2.1)-(2.3) holds. Denote by $x^{\circ}=\left(x_{1}^{\circ}, x_{2}^{\circ}\right)$ a point in $\Omega$. Then a solution $u$ to (1.7) satisfies the following properties:

(i) If

$$
u\left(x_{1}^{\circ}, x_{2}^{\circ}\right)=0, \quad \text { then } u\left(x_{1}, x_{2}^{\circ}\right)=0 \quad \forall\left(x_{1}, x_{2}^{\circ}\right) \in \Omega, \quad x_{1} \geqslant x_{1}^{\circ},
$$

(ii) If $u\left(x^{\circ}\right)>0$ then there exists a ball $B\left(x^{\circ}, \varepsilon\right)$ of centre $x^{\circ}$ and radius $\varepsilon$ in $\Omega$ such that $u>0$ in $B\left(x^{\circ}, \varepsilon\right)$. Moreover, it holds also that

$$
u>0 \quad \text { in } D_{\varepsilon}=\left\{\left(x_{1}, x_{2}\right) \in \Omega\left|x_{1} \leqslant x_{1}^{\circ},\right| x_{2}-x_{2}^{\circ} \mid<\varepsilon\right\} .
$$


Proof. Let us first show (ii). If $u\left(x^{\circ}\right)>0$ by Remark 2.3 there exists $\varepsilon$ such that

$$
u>0 \quad \text { in } B\left(x^{\circ}, \varepsilon\right) .
$$

Then from (1.7) it follows that

$$
\chi=1 \quad \text { in } B\left(x^{\circ}, \varepsilon\right) .
$$

By (2.6) one deduces that

$$
\chi=1 \quad \text { in } D_{\varepsilon} \cup B\left(x^{\circ}, \varepsilon\right) .
$$

Then (2.4) reads (recall (2.2))

$$
\nabla \cdot(K(\nabla u))=-h_{x_{1}} \leqslant 0 \quad \text { in } D_{\varepsilon} \cup B\left(x^{\circ}, \varepsilon\right) .
$$

By the maximum principle (see [12: p. 198]) it follows since $u \geqslant 0$ that if $u$ vanishes in $D_{\varepsilon} \cup B\left(x^{\circ}, \varepsilon\right)$ then $u$ vanishes identically there and this contradicts (2.12). This completes the proof of (ii).

To prove (i) it is enough to notice that if for some $x_{1}>x_{1}^{\circ}$ it holds that

$$
u\left(x_{1}, x_{2}^{\circ}\right)>0
$$

then by (ii) one would have $u\left(x_{1}^{\circ}, x_{2}^{\circ}\right)>0$ which is impossible. This completes the proof of the corollary.

For $x_{2} \in(a, b)$ we can now define

$$
\phi\left(x_{2}\right)= \begin{cases}\gamma\left(x_{2}\right) & \text { if }\left\{x_{1} \mid u\left(x_{1}, x_{2}\right)>0\right\}=\emptyset, \\ \sup \left\{x_{1} \mid u\left(x_{1}, x_{2}\right)>0\right\} & \text { else. }\end{cases}
$$

It is clear that $\phi\left(x_{2}\right)$ is well defined. Moreover, one has by (i) of Corollary 2.4 that

$$
[u>0]=\left\{\left(x_{1}, x_{2}\right) \in \Omega \mid x_{1}<\phi\left(x_{2}\right)\right\} .
$$

Our goal is now to show that the function $\phi$ is continuous. As we mentioned earlier, we suppose that $\Omega$ is piecewise smooth. In particular, we assume that

$$
\underline{\gamma}, \bar{\gamma} \text { are continuous on }(a, b) .
$$

Then we can establish the following proposition.

Proposition 2.5 Let $u$ be a solution to (1.7). Assume that (1.5), (1.6), (2.1)-(2.3), (2.15) hold. Then the function $\phi$ is lower semicontinuous on $(a, b)$.

Proof. Let $x_{2}^{\circ} \in(a, b)$ and $\lambda$ such that

$$
\lambda<\phi\left(x_{2}^{\circ}\right) .
$$

If $\underline{\gamma}\left(x_{2}^{\circ}\right)=\phi\left(x_{2}^{\circ}\right)$ then by the continuity of $\underline{\gamma}$ it holds that

$$
\lambda<\underline{\gamma}\left(x_{2}\right) \leqslant \phi\left(x_{2}\right)
$$


on a neighbourhood of $x_{2}^{\circ}$ in $(a, b)$ and the lower semicontinuity of $\phi$ follows. If now

$$
\underline{\gamma}\left(x_{2}^{\circ}\right) \leqslant \lambda<\phi\left(x_{2}^{\circ}\right)
$$

then for $\mu \in\left(\lambda, \phi\left(x_{2}^{\circ}\right)\right)$ (by (2.14)) it holds that

$$
u\left(\mu, x_{2}^{\circ}\right)>0 .
$$

Thus, by Corollary 2.4, one has (see (2.11))

$$
u>0 \quad \text { in } D_{\varepsilon} \cup B_{\varepsilon}\left(\left(\mu, x_{2}^{\circ}\right), \varepsilon\right) .
$$

Then, by (2.13),

$$
\lambda<\mu<\phi\left(x_{2}\right) \quad \text { on } \quad\left(x_{2}^{\circ}-\varepsilon, x_{2}^{\circ}+\varepsilon\right) .
$$

This shows the lower semicontinuity of $\phi$ in this case and completes the proof of the proposition.

REMARK 2.6 In order for the free boundary to be defined by a function as in (2.13), the nonnegativity of $h$ is necessary. Consider, for instance,

$$
\Omega=(0,1) \times(0,1) .
$$

Then, if $h$ denotes a smooth function in $\Omega$ such that

$$
h\left(x_{1}, x_{2}\right)= \begin{cases}1 & \text { for } \quad x_{1} \in\left(0, \frac{1}{3}\right), \\ -1 & \text { for } \quad x_{1} \in\left(\frac{2}{3}, 1\right),\end{cases}
$$

and if $K$ denotes the identity matrix then

$$
u\left(x_{1}, x_{2}\right)= \begin{cases}\frac{1}{3}-x_{1}, & x_{1} \in\left(0, \frac{1}{3}\right), \\ 0, & x_{1} \in\left(\frac{1}{3}, \frac{2}{3}\right), \\ x_{1}-\frac{2}{3}, & x_{1} \in\left(\frac{2}{3}, 1\right),\end{cases}
$$

satisfies

$$
K(\nabla u)+h \chi_{[u>0]} e_{1}=0
$$

with $e_{1}=(1,0)$. Thus $\left(u, \chi_{[u>0]}\right)$ is solution to (1.7) but the free boundary is composed of the two curves $x_{1}=\frac{1}{3}, x_{1}=\frac{2}{3}$.

\section{Upper semicontinuity of the free boundary}

Let us first start with some preliminary results (see $[6,8])$.

PROPOSITION 3.1 Let $u$ be a solution to (1.7). Denote by $x^{\circ}=\left(x_{1}^{\circ}, x_{2}^{\circ}\right)$ a point in $\Omega$ and $B\left(x^{\circ}, r\right)$ a ball of centre $x^{\circ}$ and radius $r$ in $\Omega$. Under the assumptions (2.1), (2.2) one cannot have in $B\left(x^{\circ}, r\right)$ :

$$
u\left(x_{1}, x_{2}\right)=0 \quad \text { for } x_{2}=x_{2}^{\circ}, \quad u\left(x_{1}, x_{2}\right)>0 \quad \text { for } x_{2} \neq x_{2}^{\circ} .
$$


Proof. If (3.1) holds one has $\chi=1$ a.e. in $B\left(x^{\circ}, r\right)$ so that by (2.4) one has

$$
\begin{array}{lll} 
& \nabla \cdot(K(\nabla u))+h_{x_{1}}=0 & \text { in } B\left(x_{\circ}, r\right) \\
\text { i.e. } & \nabla \cdot(K(\nabla u))=-h_{x_{1}} \leqslant 0 & \text { in } B\left(x_{\circ}, r\right) .
\end{array}
$$

Then the situation (3.1) is in contradiction with the maximum principle (see [12]).

Proposition 3.2 Let $u$ be a solution to (1.7). With the notation of the previous proposition let $B\left(x^{\circ}, r\right)$ be a ball included in $\Omega$. Under the assumptions (2.1), (2.2) one cannot have in $B\left(x^{\circ}, r\right)$

$$
u\left(x_{1}, x_{2}\right)=0 \text { for } x_{2} \geqslant x_{2}^{\circ}, \quad u\left(x_{1}, x_{2}\right)>0 \text { for } x_{2}<x_{2}^{\circ},
$$

or

$$
u\left(x_{1}, x_{2}\right)>0 \text { for } x_{2}>x_{2}^{\circ}, \quad u\left(x_{1}, x_{2}\right)=0 \text { for } x_{2} \leqslant x_{2}^{\circ} .
$$

Proof. Let us establish the result in the case of (3.2) the proof for (3.3) being identical. Denote by $B_{+}, B_{-}$the sets defined by

$$
\begin{aligned}
& B_{+}=\left\{\left(x_{1}, x_{2}\right) \in B\left(x^{\circ}, r\right) \mid x_{2}>x_{2}^{\circ}\right\}, \\
& B_{-}=\left\{\left(x_{1}, x_{2}\right) \in B\left(x^{\circ}, r\right) \mid x_{2}<x_{2}^{\circ}\right\} .
\end{aligned}
$$

Let $\xi$ be a function of $\mathcal{D}\left(B\left(x^{\circ}, r\right)\right)$. One has (if one denotes by $B$ the ball $\left.B\left(x^{\circ}, r\right)\right)$ that

$$
\begin{aligned}
0 & =\int_{B}\left\{K(\nabla u) \cdot \nabla \xi+h \chi \xi_{x_{1}}\right\} \mathrm{d} x \\
& =\int_{B_{-}}\left\{K(\nabla u) \cdot \nabla \xi+h \chi \xi_{x_{1}}\right\} \mathrm{d} x+\int_{B_{+}} h \chi \xi_{x_{1}} \mathrm{~d} x .
\end{aligned}
$$

(This follows from the facts that $\pm \xi$ is a test function for (1.7) and $u=0$ on $B_{+}$).

Now on $B_{+}$, by (2.4), it holds that

$$
\begin{gathered}
\nabla \cdot(K(\nabla u))+(h \chi)_{x_{1}}=0 \\
\text { i.e. }(h \chi)_{x_{1}}=0 .
\end{gathered}
$$

It follows that $h \chi$ is independent of $x_{1}$ in $B_{+}$. From (3.5) and since $\chi=1$ on $[u>0]$, one deduces

$$
0=\int_{B_{-}}\left\{K(\nabla u) \cdot \nabla \xi+h \xi_{x_{1}}\right\} \mathrm{d} x+\int_{B_{+}}(h \chi \xi)_{x_{1}} \mathrm{~d} x
$$

Integrating the last integral, in $x_{1}$ first, one gets since $\xi \in \mathcal{D}\left(B\left(x^{\circ}, r\right)\right)$

$$
\int_{B_{+}}(h \chi \xi)_{x_{1}} \mathrm{~d} x=0
$$

Thus, it follows from (3.6) that

$$
\begin{aligned}
0 & =\int_{B} K(\nabla u) \cdot \nabla \xi \mathrm{d} x+\int_{B_{-}}\left\{(h \xi)_{x_{1}}-h_{x_{1}} \xi\right\} \mathrm{d} x \\
& =\int_{B} K(\nabla u) \cdot \nabla \xi \mathrm{d} x-\int_{B_{-}} h_{x_{1}} \xi \mathrm{d} x .
\end{aligned}
$$


(As above, integrating the second integral in $x_{1}$ first.)

By (2.2) one gets thus

$$
\int_{B} K(\nabla u) \cdot \nabla \xi \mathrm{d} x=\int_{B_{-}} h_{x_{1}} \xi \mathrm{d} x \geqslant 0 \quad \forall \xi \in \mathcal{D}(B), \quad \xi \geqslant 0 .
$$

It follows that

$$
\nabla \cdot(K(\nabla u)) \leqslant 0 \quad \text { in } B
$$

and (3.2) is then in contradiction with the maximum principle. This completes the proof of the Proposition 3.2.

Let us now establish two preparatory lemmas. Let $x^{\circ}=\left(x_{1}^{\circ}, x_{2}^{\circ}\right), y^{\circ}=\left(y_{1}^{\circ}, y_{2}^{\circ}\right)$ be two points of $\Omega$ such that for $u$ solution to (1.7) it holds that

$$
u\left(x^{\circ}\right)=u\left(y^{\circ}\right)=0 .
$$

Without loss of generality one can assume that

$$
x_{2}^{\circ}>y_{2}^{\circ}
$$

Then, let us set

$$
m=x_{1}^{\circ} \vee y_{1}^{\circ}, \quad D=\left\{(m,+\infty) \times\left(y_{2}^{\circ}, x_{2}^{\circ}\right)\right\} \cap \Omega
$$

where $\vee$ denotes the maximum of two numbers. Then, one has the following lemma.

LEMMA 3.3 Let $\xi \in H^{1}(D) \cap L^{\infty}(D)$ such that $\xi \geqslant 0, \xi=0$ on $\left[x_{1}=m\right]$ then it holds, if $u$ denotes a solution to (1.7), that

$$
\int_{D} K(\nabla u) \cdot \nabla \xi \mathrm{d} x-\int_{D \cap[u>0]} h_{x_{1}} \xi \mathrm{d} x \leqslant 0
$$

$\left(\left[x_{1}=m\right]\right.$ denotes the set of points $\left.\left(m, x_{2}\right) \in \bar{D}\right)$.

Proof. In (1.7) let us consider the test function

$$
z=\chi_{D} H_{\delta}(u) \xi
$$

where $\chi_{D}$ denotes the characteristic function of $D, H_{\delta}$ the function defined by (2.7). It is easy to see that, by (3.9), $z$ is a suitable test function. Thus we get

$$
\int_{D}\left\{K(\nabla u) \cdot \nabla\left(H_{\delta}(u) \xi\right)+h \chi\left(H_{\delta}(u) \xi\right)_{x_{1}}\right\} \mathrm{d} x \leqslant 0 .
$$

Then

$$
\begin{aligned}
\int_{D}\left\{H_{\delta}(u) K(\nabla u) \cdot \nabla \xi\right. & +H_{\delta}^{\prime}(u) \xi K(\nabla u) \cdot \nabla u \\
& \left.+h\left(H_{\delta}(u) \xi\right)_{x_{1}}\right\} \mathrm{d} x \leqslant 0 .
\end{aligned}
$$


(We used the fact that $\chi=1$ on $[u>0]$.) The second integral being nonnegative, leads to

$$
\int_{D}\left\{H_{\delta}(u) K(\nabla u) \cdot \nabla \xi+\left(h H_{\delta}(u) \xi\right)_{x_{1}}-h_{x_{1}} H_{\delta}(u) \xi\right\} \mathrm{d} x \leqslant 0 .
$$

Integrating the second integral in the $x_{1}$ direction gives

$$
\int_{D}\left\{H_{\delta}(u) K(\nabla u) \cdot \nabla \xi-H_{\delta}(u) h_{x_{1}} \xi\right\} \mathrm{d} x \leqslant-\int_{(a, b)}\left(h H_{\delta}(u) \xi\right)\left(x_{2}, \bar{\gamma}\left(x_{2}\right)\right) \mathrm{d} x_{2} \leqslant 0 .
$$

The result then follows by letting $\delta$ go to 0 .

We then have the following lemma.

Lemma 3.4 Let $\rho \in H^{1}(D) \cap L^{\infty}(D)$ such that $\rho \geqslant 0, \rho=0$ on $\left[x_{1}=m\right]$. Then

$$
\int_{D}\left\{K(\nabla u) \cdot \nabla \rho+h \chi \rho_{x_{1}}\right\} \mathrm{d} x \leqslant 0 .
$$

Proof. For $\delta>0$ small enough one sets

$$
\alpha_{\delta}\left(x_{2}\right)=\left\{\begin{array}{lll}
\frac{x_{2}-y_{2}^{\circ}}{\delta} & \text { for } \quad x_{2} \in\left(y_{2}^{\circ}, y_{2}^{\circ}+\delta\right), \\
1 & \text { for } \quad x_{2} \in\left(y_{2}^{\circ}+\delta, x_{2}^{\circ}-\delta\right), \\
\frac{x_{2}^{\circ}-x_{2}}{\delta} & \text { for } \quad x_{2} \in\left(x_{2}^{\circ}-\delta, x_{2}^{\circ}\right) .
\end{array}\right.
$$

Then, since $\chi_{D} \alpha_{\delta} \rho$ is a test function for (1.7), we have

$$
\int_{D}\left\{K(\nabla u) \cdot \nabla \rho+h \chi \rho_{x_{1}}\right\} \mathrm{d} x \leqslant \int_{D}\left\{K(\nabla u) \cdot \nabla\left(1-\alpha_{\delta}\right) \rho+h \chi\left(\left(1-\alpha_{\delta}\right) \rho\right)_{x_{1}}\right\} \mathrm{d} x .
$$

Using the fact that $\alpha_{\delta}$ is independent of $x_{1}$ and (3.12) one obtains

$$
\begin{aligned}
\int_{D}\left\{K(\nabla u) \cdot \nabla \rho+h \chi \rho_{x_{1}}\right\} \leqslant & \int_{D \cap[u>0]} h_{x_{1}}\left(1-\alpha_{\delta}\right) \rho \mathrm{d} x \\
& +\int_{D} h \chi\left(1-\alpha_{\delta}\right) \rho_{x_{1}} \mathrm{~d} x .
\end{aligned}
$$

The result follows by letting $\delta$ go to 0 .

From now on, we make the following assumptions on the matrix $K$. We suppose $K$ smooth enough such that:

$$
k_{21} \frac{h}{k_{11}} \text { is Lipschitz continuous, nondecreasing in } x_{2},
$$

for any $\alpha>x_{1}$ the function

$$
\begin{array}{r}
k_{12} \int_{x_{1}}^{\alpha}\left(\frac{h}{k_{11}}\right)_{x_{2}}\left(\xi, x_{2}\right) \mathrm{d} \xi \text { is Lipschitz continuous } \\
\text { and nonincreasing in } x_{1},
\end{array}
$$




$$
\begin{array}{r}
k_{22} \int_{x_{1}}^{\alpha}\left(\frac{h}{k_{11}}\right)_{x_{2}}\left(\xi, x_{2}\right) \mathrm{d} \xi \text { is Lipschitz continuous } \\
\text { and nonincreasing in } x_{2},
\end{array}
$$

and for any $\alpha<x_{1}$ the function

$$
\begin{gathered}
k_{21} \int_{\alpha}^{x_{1}}\left(\frac{h}{k_{11}}\right)_{x_{2}}\left(\xi, x_{2}\right) \mathrm{d} \xi \text { is nonnegative, Lipschitz continuous } \\
\text { and nonincreasing in } x_{1}, \\
k_{22} \int_{\alpha}^{x_{1}}\left(\frac{h}{k_{11}}\right)_{x_{2}}\left(\xi, x_{2}\right) \mathrm{d} \xi \text { is Lipschitz continuous } \\
\text { and nonincreasing in } x_{2} .
\end{gathered}
$$

REMARK 3.5 In the case where

$$
\frac{h}{k_{11}}=f\left(x_{1}\right)
$$

where $f$ is a function depending on $x_{1}$ only, all the above assumptions hold true provided, for instance, that $\left(k_{21}\right)_{x_{2}} \geqslant 0$.

We can now establish the following theorem.

THEOREM 3.6 Let $u$ be a solution to (1.7). Assume that (1.5), (1.6), (2.1)-(2.3), (2.15), (3.14)(3.18) hold, then the function $\phi$ defined in (2.13) is a continuous function on $(a, b)$.

Proof. Due to Proposition 2.5 it is enough to show that $\phi$ is upper semicontinuous. Let $\varepsilon>0$ be given and let $x_{2}^{\circ} \in(a, b)$. We would like to show that

$$
E=\left\{x_{2} \mid \phi\left(x_{2}\right)<\phi\left(x_{2}^{\circ}\right)+4 \varepsilon\right\}
$$

is a neighbourhood of $x_{2}^{\circ}$-this clearly will complete the proof. If $\phi\left(x_{2}^{\circ}\right)=\bar{\gamma}\left(x_{2}^{\circ}\right)$ the result follows from the continuity of $\bar{\gamma}$ since

$$
\left\{x_{2} \mid \bar{\gamma}\left(x_{2}\right)<\bar{\gamma}\left(x_{2}^{\circ}\right)+4 \varepsilon\right\} \subset\left\{x_{2} \mid \phi\left(x_{2}\right)<\phi\left(x_{2}^{\circ}\right)+4 \varepsilon\right\}
$$

and the first of the above sets is open. So, let us suppose that

$$
\phi\left(x_{2}^{\circ}\right)<\bar{\gamma}\left(x_{2}^{\circ}\right) \quad \text { and } \quad x^{\circ}=\left(\phi\left(x_{2}^{\circ}\right), x_{2}^{\circ}\right) \in \Omega .
$$

(We will come back at the end of the proof on the case where $x^{\circ} \in \Gamma_{1}$.)

By the results of the previous section we have, of course,

$$
u\left(x_{1}, x_{2}^{\circ}\right)=0 \quad \forall x_{1} \geqslant \phi\left(x_{2}^{\circ}\right) .
$$




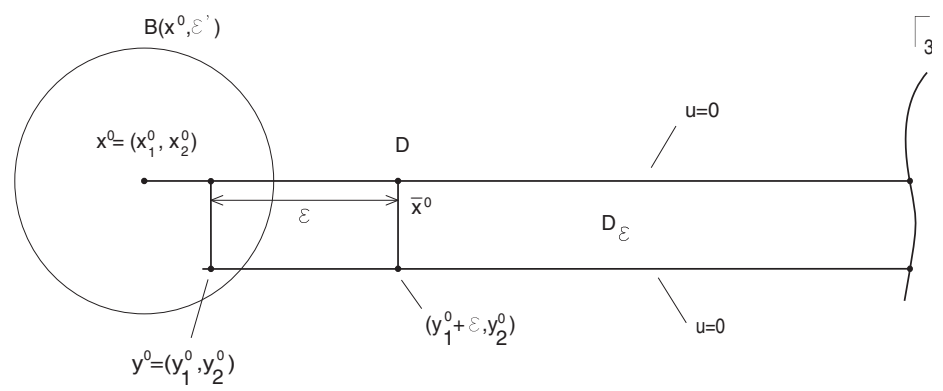

FIG. 1.

Due to the continuity of $u$ (see Corollary 2.2) there exists a ball $B\left(x^{\circ}, \varepsilon^{\prime}\right)$ in $\Omega$ with $\varepsilon^{\prime}<\varepsilon$ and such that

$$
0 \leqslant u(x) \leqslant \frac{\underline{h}}{\left|k_{11}\right|_{\infty}} \varepsilon \quad \forall x \in B\left(x^{\circ}, \varepsilon^{\prime}\right)
$$

(We have denoted by $\left|k_{11}\right|_{\infty}$ the $L^{\infty}$ norm of $k_{11}$ in $\Omega$.)

Due to (3.21), (3.1) there exists $y^{\circ} \in B\left(x^{\circ}, \varepsilon^{\prime}\right)$ such that

$$
y_{1}^{\circ}>x_{1}^{\circ}=\phi\left(x_{2}^{\circ}\right), \quad u\left(y^{\circ}\right)=0 .
$$

As we will see later, we can assume without loss of generality that

$$
y_{2}^{\circ}<x_{2}^{\circ} .
$$

The situation considered is described in Fig. 1.

Let us set

$$
\begin{gathered}
D=\left\{\left(y_{1}^{\circ},+\infty\right) \times\left(y_{2}^{\circ}, x_{2}^{\circ}\right)\right\} \cap \Omega, \\
D_{\varepsilon}=\left\{\left(y_{1}^{\circ}+\varepsilon,+\infty\right) \times\left(y_{2}^{\circ}, x_{2}^{\circ}\right)\right\} \cap \Omega .
\end{gathered}
$$

Then, we have the following lemma.

LEMMA 3.7 It holds that

$$
u=0 \quad \text { on } \quad D_{\varepsilon} .
$$

Proof. Let us introduce $q$ the function defined by

$$
q=q\left(x_{1}, x_{2}\right)= \begin{cases}\int_{x_{1}}^{y_{1}^{\circ}+\varepsilon} \frac{h}{k_{11}}\left(\xi, x_{2}\right) \mathrm{d} \xi & \text { for } x_{1}<y_{1}^{\circ}+\varepsilon \\ 0 & \text { for } x_{1} \geqslant y_{1}^{\circ}+\varepsilon .\end{cases}
$$

One has, of course, by (2.3) on the line $x_{1}=y_{1}^{\circ}$ in $B\left(x^{\circ}, \varepsilon^{\prime}\right)$ that

$$
0 \leqslant u \leqslant \frac{\underline{h}}{\left|k_{11}\right|_{\infty}} \cdot \varepsilon \leqslant \int_{y_{1}^{\circ}}^{y_{1}^{\circ}+\varepsilon} \frac{h}{k_{11}}\left(\xi, x_{2}\right) \mathrm{d} \xi=q .
$$


Since also $u$ vanishes on the lateral boundary of $D$ it is clear that

$$
\chi_{D}(u-q)^{+}
$$

is a test function for (1.7). ( $)^{+}$denotes the positive part of a function. So, one gets

$$
\int_{D}\left\{K(\nabla u) \cdot \nabla(u-q)^{+}+h \chi(u-q)_{x_{1}}^{+}\right\} \mathrm{d} x \leqslant 0
$$

which leads to

$$
\int_{D}\left\{K\left(\nabla(u-q)^{+}\right) \cdot \nabla(u-q)^{+}+K(\nabla q) \cdot \nabla(u-q)^{+}+h \chi(u-q)_{x_{1}}^{+}\right\} \mathrm{d} x \leqslant 0 .
$$

Using the definition of $q$ one deduces easily that

$$
\begin{aligned}
& \int_{D} K\left(\nabla(u-q)^{+}\right) \cdot \nabla(u-q)^{+} \mathrm{d} x \\
& +\int_{D \backslash D_{\varepsilon}}\left\{-h+k_{12} \int_{x_{1}}^{y_{1}^{\circ}+\varepsilon}\left(\frac{h}{k_{11}}\right)_{x_{2}}\left(\xi, x_{2}\right) \mathrm{d} \xi\right\}(u-q)_{x_{1}}^{+} \mathrm{d} x \\
& +\int_{D \backslash D_{\varepsilon}}\left\{-\frac{k_{21} h}{k_{11}}+k_{22} \int_{x_{1}}^{y_{1}^{\circ}+\varepsilon}\left(\frac{h}{k_{11}}\right)_{x_{2}}\left(\xi, x_{2}\right) \mathrm{d} \xi\right\}(u-q)_{x_{2}}^{+} \mathrm{d} x \\
& +\int_{D} h \chi(u-q)_{x_{1}}^{+} \mathrm{d} x \leqslant 0 .
\end{aligned}
$$

Since $(u-q)^{+}$vanishes on the horizontal parts of the boundary of $D$, one has by (3.14), (3.16):

$$
\begin{aligned}
& \int_{D \backslash D_{\varepsilon}}-\frac{k_{21} h}{k_{11}}(u-q)_{x_{2}}^{+} \mathrm{d} x=\int_{D \backslash D_{\varepsilon}}\left(\frac{k_{21} h}{k_{11}}\right)_{x_{2}}(u-q)^{+} \mathrm{d} x \geqslant 0 \\
& \int_{D \backslash D_{\varepsilon}}\left\{k_{22} \int_{x_{1}}^{y_{1}^{\circ}+\varepsilon}\left(\frac{h}{k_{11}}\right)_{x_{2}}\left(\xi, x_{2}\right) \mathrm{d} \xi\right\}(u-q)_{x_{2}}^{+} \mathrm{d} x \\
= & -\int_{D \backslash D_{\varepsilon}}\left\{k_{22} \int_{x_{1}}^{y_{1}^{\circ}+\varepsilon}\left(\frac{h}{k_{11}}\right)_{x_{2}}\left(\xi, x_{2}\right) \mathrm{d} \xi\right\}_{x_{2}}(u-q)^{+} \mathrm{d} x \geqslant 0 .
\end{aligned}
$$

Moreover, integrating in $x_{1}$ first, one gets from (3.15):

$$
\begin{aligned}
& \int_{D \backslash D_{\varepsilon}}\left\{k_{12} \int_{x_{1}}^{y_{1}^{\circ}+\varepsilon}\left(\frac{h}{k_{11}}\right)_{x_{2}}\left(\xi, x_{2}\right) \mathrm{d} \xi\right\}(u-q)_{x_{1}}^{+} \mathrm{d} x \\
= & -\int_{D \backslash D_{\varepsilon}}\left\{k_{12} \int_{x_{1}}^{y_{1}^{\circ}+\varepsilon}\left(\frac{h}{k_{11}}\right)_{x_{2}}\left(\xi, x_{2}\right) \mathrm{d} \xi\right\}_{x_{1}}(u-q)^{+} \mathrm{d} x \geqslant 0 .
\end{aligned}
$$

Thus from (3.29)-(3.32) one deduces

$$
\begin{aligned}
\int_{D} K\left(\nabla(u-q)^{+}\right) \cdot \nabla(u-q)^{+} \mathrm{d} x & -\int_{D \backslash D_{\varepsilon}} h(u-q)_{x_{1}}^{+} \mathrm{d} x \\
& +\int_{D} h \chi(u-q)_{x_{1}}^{+} \mathrm{d} x \leqslant 0 .
\end{aligned}
$$


Since $\chi=1$ on $[u>0], \chi(u-q)_{x_{1}}^{+}=(u-q)_{x_{1}}^{+}$and the above inequality leads to (recall that $q=0$ on $D_{\varepsilon}$ )

$$
\int_{D} K\left(\nabla(u-q)^{+}\right) \cdot \nabla(u-q)^{+} \mathrm{d} x+\int_{D_{\varepsilon}} h \chi u_{x_{1}} \mathrm{~d} x \leqslant 0 .
$$

Let us consider now the function $\rho$ defined by

$$
\rho=\rho\left(x_{1}, x_{2}\right)= \begin{cases}0 & \text { for } x_{1}<y_{1}^{\circ}+\varepsilon \\ \int_{y_{1}^{\circ}+\varepsilon}^{x_{1}} \frac{h}{k_{11}}\left(\xi, x_{2}\right) \mathrm{d} \xi & \text { for } x_{1} \geqslant y_{1}^{\circ}+\varepsilon\end{cases}
$$

Applying Lemma 3.4 it holds that

$$
\int_{D}\left\{K(\nabla u) \nabla \rho+h \chi \rho_{x_{1}}\right\} \mathrm{d} x \leqslant 0
$$

This reads

$$
\int_{D_{\varepsilon}}\left\{\left(k_{11} u_{x_{1}}+k_{12} u_{x_{2}}\right) \frac{h}{k_{11}}+\left(k_{21} u_{x_{1}}+k_{22} u_{x_{2}}\right) \int_{y_{1}^{\circ}+\varepsilon}^{x_{1}}\left(\frac{h}{k_{11}}\right)_{x_{2}}\left(\xi, x_{2}\right) \mathrm{d} \xi+\frac{h^{2} \chi}{k_{11}}\right\} \mathrm{d} x \leqslant 0 .
$$

By (3.17), (3.18) we get

$$
\begin{aligned}
& \int_{D_{\varepsilon}}\left\{k_{21} \int_{y_{1}^{\circ}+\varepsilon}^{x_{1}}\left(\frac{h}{k_{11}}\right)_{x_{2}}\left(\xi, x_{2}\right) \mathrm{d} \xi\right\} u_{x_{1}} \mathrm{~d} x \\
= & -\int_{D_{\varepsilon}}\left\{k_{21} \int_{y_{1}^{\circ}+\varepsilon}^{x_{1}}\left(\frac{h}{k_{11}}\right)_{x_{2}}\left(\xi, x_{2}\right) \mathrm{d} \xi\right\}_{x_{1}} u \mathrm{~d} x \\
+ & \int_{\Gamma_{3}}\left\{k_{21} \int_{y_{1}^{\circ}+\varepsilon}^{x_{1}}\left(\frac{h}{k_{11}}\right)_{x_{2}}\left(\xi, x_{2}\right) \mathrm{d} \xi\right\} u \mathrm{~d} x_{2} \geqslant 0, \\
& \int_{D_{\varepsilon}}\left\{k_{22} \int_{y_{1}^{\circ}+\varepsilon}^{x_{1}}\left(\frac{h}{k_{11}}\right)_{x_{2}}\left(\xi, x_{2}\right) \mathrm{d} \xi\right\} u_{x_{2}} \mathrm{~d} x \\
= & -\int_{D_{\varepsilon}}\left\{k_{22} \int_{y_{1}^{\circ}+\varepsilon}^{x_{1}}\left(\frac{h}{k_{11}}\right)_{x_{2}}\left(\xi, x_{2}\right) \mathrm{d} \xi\right\}_{x_{2}} u \mathrm{~d} x \geqslant 0 .
\end{aligned}
$$

Thus (3.35) reads (recall that $\chi \nabla u=\nabla u$ and (3.14))

$$
\begin{aligned}
& \int_{D_{\varepsilon}} \chi\left\{h u_{x_{1}}+\frac{k_{12} h}{k_{11}} u_{x_{2}}+\frac{k_{21} h}{k_{11}} u_{x_{2}}+\frac{h^{2}}{k_{11}}\right\} \mathrm{d} x \\
\leqslant & \int_{D_{\varepsilon}} \frac{k_{21} h}{k_{11}} u_{x_{2}} \mathrm{~d} x \leqslant 0 .
\end{aligned}
$$


Combining (3.33) and (3.36) we obtain easily:

$$
\begin{aligned}
& \int_{D \backslash D_{\varepsilon}} K\left(\nabla(u-q)^{+}\right) \cdot \nabla(u-q)^{+} \mathrm{d} x \\
+ & \int_{D_{\varepsilon}} \chi\left\{k_{11} u_{x_{1}}^{2}+\left(k_{12}+k_{21}\right) u_{x_{1}} u_{x_{2}}+k_{22} u_{x_{2}}^{2}\right. \\
+ & \left.2 h u_{x_{1}}+\left(k_{12}+k_{21}\right) \frac{h}{k_{11}} u_{x_{2}}+\frac{h^{2}}{k_{11}}\right\} \mathrm{d} x \leqslant 0 .
\end{aligned}
$$

The integrand in the second integral above is equal to

$$
\begin{aligned}
& \chi\left\{k_{11}\left(u_{x_{1}}^{2}+\frac{2 h}{k_{11}} u_{x_{1}}+\frac{h^{2}}{k_{11}^{2}}\right)+\left(k_{12}+k_{21}\right) u_{x_{2}}\left(u_{x_{1}}+\frac{h}{k_{11}}\right)+k_{22} u_{x_{2}}^{2}\right\} \\
= & \chi\left\{k_{11}\left(u_{x_{1}}+\frac{h}{k_{11}}\right)^{2}+\left(k_{12}+k_{21}\right) u_{x_{2}}\left(u_{x_{1}}+\frac{h}{k_{11}}\right)+k_{22} u_{x_{2}}^{2}\right\} \geqslant 0
\end{aligned}
$$

since the matrix $K$ is positive definite. Thus from (3.37) one derives that

$$
(u-q)^{+}=0 \quad \text { on } D \backslash D_{\varepsilon} .
$$

This implies that $u=0$ on $D_{\varepsilon}$ and completes the proof of the lemma.

Due to the lemma we have now shown that

$$
\phi\left(x_{2}\right) \leqslant y_{1}^{\circ}+\varepsilon \leqslant \phi\left(x_{2}^{\circ}\right)+2 \varepsilon \quad \forall x_{2} \in\left[y_{2}^{\circ}, x_{2}^{\circ}\right]
$$

that is to say (see (3.19)) that

$$
\left[y_{2}^{\circ}, x_{2}^{\circ}\right] \subset E .
$$

Next one sets (see Fig. 1)

$$
\bar{x}^{\circ}=\left(y_{1}^{\circ}+\varepsilon, x_{2}^{\circ}\right)
$$

and one uses this point as the point $x^{\circ}$ before. That is to say, one can find a ball $B\left(\bar{x}^{\circ}, \varepsilon^{\prime}\right)$ in $\Omega$ such that $\varepsilon^{\prime}<\varepsilon$ and

$$
0 \leqslant u(x) \leqslant \frac{\underline{h}}{\left|k_{11}\right|_{\infty}} \varepsilon \quad \forall x \in B\left(\bar{x}^{\circ}, \varepsilon^{\prime}\right) .
$$

Moreover (see Proposition 3.2) one can find

$$
\bar{y}^{\circ}=\left(\bar{y}_{1}^{\circ}, \bar{y}_{2}^{\circ}\right) \in B\left(\bar{x}^{\circ}, \varepsilon^{\prime}\right)
$$

such that

$$
\bar{y}_{2}^{\circ}>x_{2}^{\circ}, \bar{y}_{1}^{\circ}>y_{1}^{\circ}+\varepsilon, \quad u\left(\bar{y}^{\circ}\right)=0 .
$$

Then, arguing as above, one can easily show that

$$
u=0 \quad \text { on }\left(\bar{y}_{1}^{\circ}+\varepsilon,+\infty\right) \times\left(x_{2}^{\circ}, \bar{y}_{2}^{\circ}\right) \cap \Omega .
$$


(Recall that $u\left(x_{1}, \bar{y}_{2}^{\circ}\right)=0 \forall x_{1} \geqslant \bar{y}_{1}^{\circ}$ ).

Then for $x_{2} \in\left[x_{2}^{\circ}, \bar{y}_{2}^{\circ}\right]$ it holds that

$$
\phi\left(x_{2}\right)<\bar{y}_{1}^{\circ}+2 \varepsilon<\phi\left(x_{2}^{\circ}\right)+4 \varepsilon
$$

that is to say we have now proved that

$$
\left(y_{2}^{\circ}, \bar{y}_{2}^{\circ}\right) \subset E
$$

and the upper semicontinuity of $\phi$ at $x_{2}^{\circ}$. Clearly a choice of $y^{\circ}$ above $x^{\circ}$ (see (3.24)) could have been handled the same way. If now $x^{\circ}$ in (3.20) belongs to $\Gamma_{1}$ then one applies the above technique to

$$
\tilde{x}^{\circ}=\left(\phi\left(x_{2}^{\circ}\right)+\varepsilon, x_{2}^{\circ}\right)
$$

to conclude. This completes the proof of the theorem.

REMARK 3.8 It is possible to weaken the assumption (2.15). Indeed, we can assume only that $\gamma$ is lower semicontinuous and $\bar{\gamma}$ upper semicontinuous. $\Omega$ is then defined as the interior of the set given in (1.1).

REMARK 3.9 One can in fact show that $\chi$ is a characteristic function, i.e. one has

$$
\chi=\chi[u>0] .
$$

Indeed, consider for instance $C$ a connected component of the set

$$
\left\{\left(x_{1}, x_{2}\right) \in \Omega \mid \phi\left(x_{2}\right)<x_{1}<\bar{\gamma}\left(x_{2}\right)\right\} .
$$

One has $u=0$ on $C$ and thus by (2.4)

$$
(h \chi)_{x_{1}}=0 .
$$

It follows that in $C, h \chi$ is a function depending on $x_{2}$ only. Consider then a function $z \in H^{1}(\Omega)$, vanishing outside $C$ and in a neighbourhood of $\partial C \cap \Omega$. One has, by (1.7), if $z$ is nonnegative on $\Gamma_{3}$, that

$$
\int_{C}(h \chi z)_{x_{1}} \mathrm{~d} x=\int_{a}^{b} h \chi z\left(\bar{\gamma}\left(x_{2}\right), x_{2}\right) \mathrm{d} x_{2} \leqslant 0 .
$$

This implies that $h \chi=0$ in $C$ and thus $\chi=0$ in $C$.

\section{Applications}

\subsection{The dam problem}

We refer the reader to $[1,5,8]$ for details on the problem. We just describe it briefly. We consider a bidimensional porous medium given as in Fig. 2.

In Fig. 2, $\Gamma_{3}=\Gamma_{31} \cup \Gamma_{32}$ where $\Gamma_{31}$ is the part of the dam covered by the atmosphere, $\Gamma_{32}$ the part covered by water. $\Gamma_{1} \cup \Gamma_{2}$ is an impervious boundary for the medium (for instance). $D$ is the 


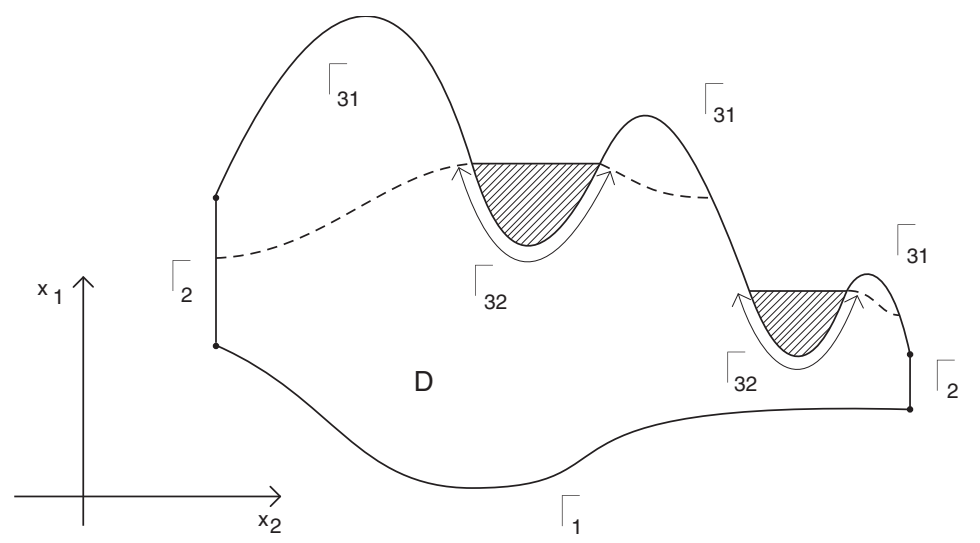

FIG. 2.

medium. Then a solution of the filtration problem through this porous medium (see $[1,5,8]$ ) is a couple of functions $(u, \chi)$ such that

$$
\left\{\begin{array}{l}
(u, \chi) \in H^{1}(D) \times L^{\infty}(D), \quad 0 \leqslant \chi \leqslant 1 \text { a.e. in } D, \\
u \geqslant 0 \text { a.e. in } D, \quad u=\varphi \text { on } \Gamma_{3}, \quad \chi=1 \text { on }[u>0], \\
\int_{D}\left\{\nabla u \cdot \nabla \xi+\chi \xi_{x_{1}}\right\} \mathrm{d} x \leqslant 0 \quad \forall \xi \in H^{1}(D), \quad \xi=0 \text { on } \Gamma_{32}, \quad \xi \geqslant 0 \text { on } \Gamma_{31} .
\end{array}\right.
$$

$u$ is the hydrostatic pressure in the medium, $\varphi$ the outside pressure- - for details see the literature quoted in [8] or [11].

Suppose that $\Gamma_{1}, \Gamma_{3}$ are the graphs of function $\gamma, \bar{\gamma}$ defined on some interval. Consider a connected component of $\Gamma_{31}$. This is the graph of the function $\bar{\gamma}$ on some interval $(a, b)$. Define then $\Omega$ as in (1.1) and denote by $\Gamma_{1}, \Gamma_{2}, \Gamma_{3}$ the different parts of the boundary of $\Omega$ as in (1.2)-(1.4). Then clearly if $z \in H^{1}(\Omega), z=0$ on $\Gamma_{1} \cup \Gamma_{2}, z \geqslant 0$ on $\Gamma_{3}$

is a test function for (4.1) and it holds that

$$
\left\{\begin{array}{l}
(u, \chi) \in H^{1}(\Omega) \times L^{\infty}(\Omega), \quad 0 \leqslant \chi \leqslant 1 \text { a.e. in } \Omega, \\
u \geqslant 0 \text { a.e. in } \Omega, \quad \chi=1 \text { on }[u>0], \\
\int_{\Omega}\left\{\nabla u \nabla z+\chi z_{x_{1}}\right\} \mathrm{d} x \leqslant 0 \quad \forall z \in H^{1}(\Omega), \quad z=0 \text { on } \Gamma_{1} \cup \Gamma_{2}, \quad z \geqslant 0 \text { on } \Gamma_{3} .
\end{array}\right.
$$

So, we see that $(u, \chi)$ is solution to (1.7) with $K$ the identity matrix, $h=1$. Thanks to Remark 3.5, Theorem 3.6 applies and the free boundary is continuous on $(a, b)$.

REMARK 4.1 One can easily show that $\phi=\bar{\gamma}$ under the boundary parts $\Gamma_{32}$.

REMARK 4.2 It is of course possible to handle more general cases including the case of a variable permeability matrix $K$ or leaky boundary conditions. The reader is referred for instance to [9]. 


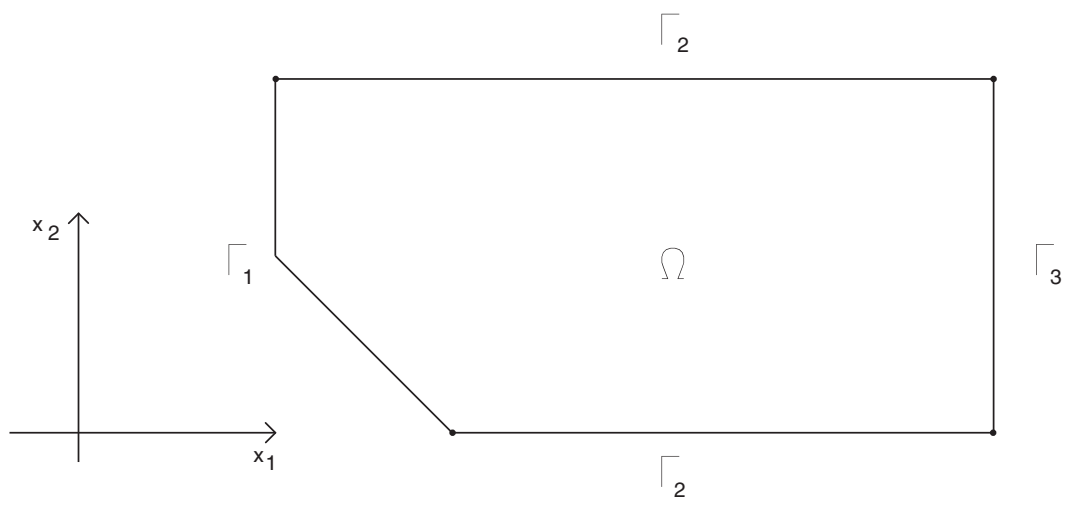

FIG. 3.

\subsection{A problem in aluminium electrolysis}

If $\Omega$ is the section of an aluminium electrolytic cell (see Fig. 3) one is led to the following problem.

Find $(T, q)$ solution to

$$
\left\{\begin{array}{l}
(T, q) \in H^{1}(\Omega) \times L^{\infty}(\Omega), \quad 0 \leqslant q \leqslant 1 \text { a.e. in } \Omega, \\
T-T_{s} \leqslant 0 \text { a.e. in } \Omega, \quad q=0 \text { on }\left[T<T_{s}\right], \\
\int_{\Omega} k \nabla T \cdot \nabla z \mathrm{~d} x+\int_{\Omega} h q z_{x_{1}} \mathrm{~d} x \quad+\int_{\Gamma_{1}} \alpha\left(T-T_{c}\right) z \mathrm{~d} \sigma=\int_{\Gamma_{3}} h z \mathrm{~d} \sigma \quad \forall z \in H^{1}(\Omega) .
\end{array}\right.
$$

$k$ is the diffusion coefficient supposed to satisfy $k \geqslant \underline{K}>0 . T$ is the temperature in the electrolytic bath and $T_{s}$ is the solidification temperature. $\alpha, T_{c}$ are some constants, $\mathrm{d} \sigma$ is the surface measure on the boundary of $\Omega, h$ is a function of $x_{2}$ only satisfying (1.6), (2.3). This problem was introduced in [13] and [3] and we refer the reader to those references for the physical set-up of (4.4). Modulo the fact that the free boundary is of measure 0 the authors of [3] establish the existence and uniqueness of a solution to (4.4)—see also [13]. We would like to show that this is indeed the case and that the free boundary is a continuous function: of course, by free boundary we mean (with the same precautions as before) the set

$$
\overline{\left[T<T_{S}\right]} \cap \overline{\left[T=T_{S}\right]} .
$$

For this purpose let us reformulate the problem by setting

$$
u=T_{s}-T, \chi=1-q .
$$

Noting that

$$
\int_{\Omega} h z_{x_{1}}=\int_{\Gamma_{3}} h z \mathrm{~d} \sigma+\int_{\Gamma_{1}} h z v_{x_{1}} \mathrm{~d} \sigma
$$

where $v_{x_{1}}$ denotes the first component of the outward unit normal to $\Gamma_{1}$, the last equation of (4.4) reads

$$
\int_{\Omega} k \nabla T \cdot \nabla z \mathrm{~d} x-\int_{\Omega} h(1-q) z_{x_{1}} \mathrm{~d} x=-\int_{\Gamma_{1}}\left\{h v_{x_{1}}+\alpha\left(T-T_{c}\right)\right\} z \mathrm{~d} \sigma \quad \forall z \in H^{1}(\Omega) .
$$


So, one has also

$$
\int_{\Omega} k \nabla\left(T-T_{s}\right) \cdot \nabla z \mathrm{~d} x-\int_{\Omega} h(1-q) z_{x_{1}} \mathrm{~d} x=-\int_{\Gamma_{1}}\left\{h v_{x_{1}}+\alpha\left(T-T_{c}\right)\right\} z \mathrm{~d} \sigma \quad \forall z \in H^{1}(\Omega) .
$$

Thus, from this equality one sees that $(u, \chi)$ satisfies

$$
\left\{\begin{array}{l}
(u, \chi) \in H^{1}(\Omega) \times L^{\infty}(\Omega), \quad 0 \leqslant \chi \leqslant 1 \text { a.e. in } \Omega, \\
u \geqslant 0, \quad \chi=1 \text { on }[u>0], \\
\int_{\Omega}\left\{k \nabla u \cdot \nabla z+h \chi z_{x_{1}}\right\} \mathrm{d} x=0 \quad \forall z \in H^{1}(\Omega), \quad z=0 \text { on } \Gamma_{1} .
\end{array}\right.
$$

Thus $(u, \chi)$ is solution to (1.7). Assuming

$$
h=h\left(x_{2}\right) \in L^{\infty}(\Omega) \cap H^{1}(\Omega), \quad h \geqslant \underline{h}>0
$$

one has (1.6) and (2.2), (2.3). Assuming

$$
k \in L^{\infty}(\Omega), \quad k \geqslant \underline{K}>0
$$

one has (1.5), (2.1). So, provided that for $\alpha>x_{1}, h, k$ are smooth and such that

$$
\begin{array}{r}
k \int_{x_{1}}^{\alpha}\left(\frac{h}{k}\right)_{x_{2}}\left(\xi, x_{2}\right) \mathrm{d} \xi \text { is Lipschitz continuous } \\
\text { nonincreasing in } x_{2}
\end{array}
$$

and for $\alpha<x_{1}$

$$
\begin{array}{r}
k \int_{\alpha}^{x_{1}}\left(\frac{h}{k}\right)_{x_{2}}\left(\xi, x_{2}\right) \mathrm{d} \xi \text { is Lipschitz continuous } \\
\text { and nonincreasing in } x_{2} .
\end{array}
$$

One can apply Theorem 3.6 and conclude to the continuity of the free boundary. For (4.10), (4.11) to hold we recall also the Remark 3.5.

\section{Acknowledgement}

This work has been supported by the Swiss National Science Foundation under the contract \#2149278.96.

\section{REFERENCES}

1. Alt, H. W. Strömungen durch inhomogene poröse medien mit freiem rand. J. Reine Ange. Math. 305, (1979) 89-115.

2. BAIOCCHI, C. Su un problema di frontiera libera connesso a questioni di idraulica. Am. Mat. Pura Appl. 92, (1972) 107-127.

3. BermúdeZ, A., MuÑIZ, M. C., \& Quintela, P. Existence and uniqueness for a free boundary problem in aluminium electrolysis. J. Math. Anal. Appl 191, (1995) 497-527.

4. Brezis, H. Analyse Fonctionnelle-Théorie et Applications. Masson, Paris (1983). 
5. Brezis, H., Kinderlehrer, D., \& Stampacchia, G. Sur une nouvelle formulation du problème de l'écoulement à travers une digue. C.R. Acad. Sc., Paris, A 287, (1978) 711-714.

6. Carrillo, J. \& Chipot, M. On the dam problem. J. Diff. Eqns 45, (1982) 234-271.

7. Carrillo, J. \& Chipot, M. The dam problem with leaky boundary conditions. Appl. Math. Optim. 28, (1993) 57-85.

8. Chipot, M. Variational Inequalities and Flow in Porous Media, Appl. Math. Science Series, vol. 52. Springer, NewYork (1984).

9. Chipot, M. \& Lyaghfouri, A. The dam problem for linear Darcy's law and nonlinear leaky boundary conditions. Adv. Diff. Eqns. 3(1), (1998) 1-50.

10. Dautray, R. \& Lions, J. L. Mathematical Analysis and Numerical Methods for Science and Technology, vol. 1. Springer, NewYork (1990).

11. Friedman, A. Variational Principles and Free-Boundary Problems. R.E. Krieger, Malabar Fl, (1988).

12. Gilbarg, D. \& Trudinger, N. S. Elliptic Partial Differential Equations of Second Order. Springer, New York (1985)

13. MuÑIZ, M. C. Estudio matemático de un problema de Stefan relacionado con la modelización termoeléctrica de cubas de electrolisis de aluminio, Thesis, Universidade de Santiago de Compostela, (1995).

14. Rodrigues, J. F. Obstacle Problems in Mathematical Physics, North-Holland Mathematics Studies, vol. 134. North-Holland, Australia (1987). 